\title{
Gut bacterial deamination of residual levodopa medication for Parkinson's disease
}

\author{
Sebastiaan P. van Kessel ${ }^{1}$, Hiltje R. de Jong ${ }^{1}$, Simon L. Winkel', Sander S. van Leeuwen ${ }^{1,2}$, Sieger A. Nelemans ${ }^{3}$, \\ Hjalmar Permentier ${ }^{4}$, Ali Keshavarzian ${ }^{5}$ and Sahar El Aidy ${ }^{1 *}$ (I)
}

\begin{abstract}
Background: Parkinson's disease (PD) is a progressive neurodegenerative disorder characterized by both motor and non-motor symptoms. Gastrointestinal tract dysfunction is one of the non-motor features, where constipation is reported as the most common gastrointestinal symptom. Aromatic bacterial metabolites are attracting considerable attention due to their impact on gut homeostasis and host's physiology. In particular, Clostridium sporogenes is a key contributor to the production of these bioactive metabolites in the human gut.

Results: Here, we show that C. sporogenes deaminates levodopa, the main treatment in Parkinson's disease, and identify the aromatic aminotransferase responsible for the initiation of the deamination pathway. The deaminated metabolite from levodopa, 3-(3,4-dihydroxyphenyl)propionic acid, elicits an inhibitory effect on ileal motility in an ex vivo model. We detected 3-(3,4-dihydroxyphenyl)propionic acid in fecal samples of Parkinson's disease patients on levodopa medication and found that this metabolite is actively produced by the gut microbiota in those stool samples.

Conclusions: Levodopa is deaminated by the gut bacterium C. sporogenes producing a metabolite that inhibits ileal motility ex vivo. Overall, this study underpins the importance of the metabolic pathways of the gut microbiome involved in drug metabolism not only to preserve drug effectiveness, but also to avoid potential side effects of bacterial breakdown products of the unabsorbed residue of medication.
\end{abstract}

Keywords: Non-motor symptoms, Gastrointestinal motility, Clostridium sporogenes, Drug side effects, Bioactive metabolites, Aminotransferase

\section{Background}

Gut bacteria produce a wide range of small bioactive molecules from different chemical classes, including aromatic amino acids [1]. Bacterial products from aromatic amino acid degradation have been shown to play a critical role in intestinal barrier function, immune modulation, and gut motility [2-6]. In the lower part of the gastrointestinal

\footnotetext{
* Correspondence: sahar.elaidy@rug.nl

'Department of Molecular Immunology and Microbiology, Groningen Biomolecular Sciences and Biotechnology Institute (GBB), University of Groningen, Nijenborgh 7, 9747 AG Groningen, The Netherlands

Full list of author information is available at the end of the article
}

(GI) tract, where oxygen is limited, aromatic amino acid degradation by anaerobic bacteria involves reductive or oxidative deamination [7] resulting in production of aromatic metabolites [8-11]. Although the enzymes involved in the deamination pathway of the aromatic amino acids tryptophan, phenylalanine, and tyrosine have been described [11-13], the enzyme involved in the initial transamination step remains unknown.

Recently, small intestinal (SI) microbiota have been implicated in the interference with levodopa drug availability $[14,15]$. Early in vivo studies showed that 90\%

(c) The Author(s). 2020 Open Access This article is licensed under a Creative Commons Attribution 4.0 International License, which permits use, sharing, adaptation, distribution and reproduction in any medium or format, as long as you give appropriate credit to the original author(s) and the source, provide a link to the Creative Commons licence, and indicate if changes were made. The images or other third party material in this article are included in the article's Creative Commons licence, unless indicated otherwise in a credit line to the material. If material is not included in the article's Creative Commons licence and your intended use is not permitted by statutory regulation or exceeds the permitted use, you will need to obtain permission directly from the copyright holder. To view a copy of this licence, visit http://creativecommons.org/licenses/by/4.0/. The Creative Commons Public Domain Dedication waiver (http://creativecommons.org/publicdomain/zero/1.0/) applies to the data made available in this article, unless otherwise stated in a credit line to the data. 
of levodopa is transported to the circulatory system [1618 ], leaving a $\sim 10 \%$ unabsorbed fraction of residual levodopa that can act as substrate for other bacterial species associated with the lower, more anaerobic regions of the GI tract [19]. Such bacterial-residual drug interaction might act as bioactive metabolites with an impact on gut homeostasis.

Parkinson's disease (PD) is often associated with nonmotor symptoms especially in the GI tract. GI tract dysfunction such as constipation, drooling, and swallowing disorders occurs frequently in PD patients, especially constipation, which is reported in $80-90 \%$ of the PD patients [20]. Importantly, chronic idiopathic constipation is associated with SI motor abnormalities in the esophagus, stomach, jejunum, and ileum [21, 22] and patients with constipation have a longer SI transit time compared to controls [22]. Only recently, SI dysfunction in PD was studied showing that the transit time in the SI was significantly longer in PD patients compared to healthy controls (HC) $[23,24]$. Using wireless electromagnetic capsules, the SI transit time was reported to be significantly higher in PD patients (400 min; $n=22$ ) compared to HC (295 $\min , n=15)$ [24].

This study uncovers the aminotransferase responsible for initiating the deamination pathway involved in the transamination of (among others) levodopa and shows that $C$. sporogenes can effectively deaminate levodopa to 3-(3,4-dihydroxyphenyl)propionic acid through the aromatic amino acid deamination pathway [11]. We show that the deamination product of gut bacterial degradation of the unabsorbed residues of levodopa in fecal samples from PD patients reduces ileal motility ex vivo. Our results highlight the urgency for further research on the effects of bacterial conversion of the unabsorbed residues of medication, which may affect host physiology.

\section{Results \\ Clostridium sporogenes deaminates levodopa through its deamination pathway}

C. sporogenes is able to deaminate proteinogenic aromatic amino acids (PAAAs) through an anaerobic deamination pathway (Fig. 1a) [11-13]. We hypothesized that levodopa, a non-proteinogenic amino acid (NPAA A) and the main treatment in PD, could be deaminated through the same pathway. Together with another NPAAA, 5-hydroxytryptophan (5-HTP, precursor of serotonin, over-the-counter available drug used to treat depression, obesity, insomnia, and chronic headaches [25]), as an analogous control compound derived from tryptophan, we screened for deamination of these compounds in batch cultures of $C$. sporogenes. Cultures were incubated with $100 \mu \mathrm{M}$ levodopa or 5 -HTP in combination with PAAAs from the growth medium and were followed over a period of $48 \mathrm{~h}$. Analysis of the samples using high-pressure liquid chromatography (HPLC) coupled to an electrochemical detector (ED) revealed that levodopa is completely converted within $24 \mathrm{~h}$ to a new metabolite, which was identified by ${ }^{1} \mathrm{H} /{ }^{13} \mathrm{C}$-NMR and LC-MS as 3-(3,4-dihydroxyphenyl)propionic acid, DHPPA (Fig. 1b, c; Additional File 1: Fig. S1A, 1B, 1C). Furthermore, the incubations showed that the PAAAs available from the growth medium did not prevent the deamination of levodopa and that, during the incubation for $48 \mathrm{~h}$, DHPPA remained stable. Similarly, 5-HTP was converted into two new unknown peaks (Additional File 1: Fig. S2A, S2B), albeit to a much lesser extent compared to levodopa. Only the first peak could be detected and assigned by LC-MS as 5-hydroxyindole-3-lactic acid (5-HILA) by its predicted exact mass (Additional File 1: Fig. S2C). The other peak is potentially 5 -hydroxyindole3-propionic acid (5-HIPA), described below.

To further investigate the involvement of the deamination pathway in levodopa and 5-HTP deamination, the enzyme responsible for the dehydratase reaction (encoded by the fldC gene [11-13]) was disrupted using the ClosTron mutagenesis system (Additional File 1: Fig. S2D) [26]. The resulting strain C. sporogenes Ll.LtrB-ery ${ }^{R} \Omega$ fldC $\left(C S^{\Omega f l d C}\right)$ was incubated with levodopa, and the PAAAs from the growth medium. Tryptophan and tyrosine were converted to their intermediates ILA (indole-3-lactic acid) and 4-HPLA (3-(4-hydroxyphenyl)lactic acid), respectively, as previously shown [11]. Analogous to tryptophan and tyrosine, levodopa was no longer deaminated to DHPPA but to its intermediate product 3-(3,4-dihydroxyphenyl)lactic acid (DHPLA) (Fig. 1d, e). Only a slight production of 4-HPPA (from tyrosine) is observed after $48 \mathrm{~h}$, presumably because of the substitution of FldABC by the similar HadABC proteins from the had-operon in C. sporogenes $[11,12]$. HPLC-ED analysis of the 5-HILA production from 5 -HTP by the fldC mutant was hampered by the production of coeluting 4-HPLA, the intermediate deamination product produced from tyrosine (described above). However, the analysis revealed that the second unknown peak produced from 5-HTP was no longer produced by $C^{\Omega f l d C}$ (Additional File 1: Fig. S2E, S2F), demonstrating that 5-HTP conversion is affected and suggesting that the unknown product is 5-HIPA. Overall, the results show that the deamination pathway from C. sporogenes not only is involved in the deamination of PAAAs but also is in the deamination of the NPAAAs, levodopa and 5-HTP.

\section{Identification of the aromatic aminotransferase responsible for initiation of the deamination pathway}

The aromatic aminotransferase responsible for the transamination of levodopa and the other (N)PAAAs is crucial for the initiation of the reductive deamination pathway and for the full deamination of the substrates 


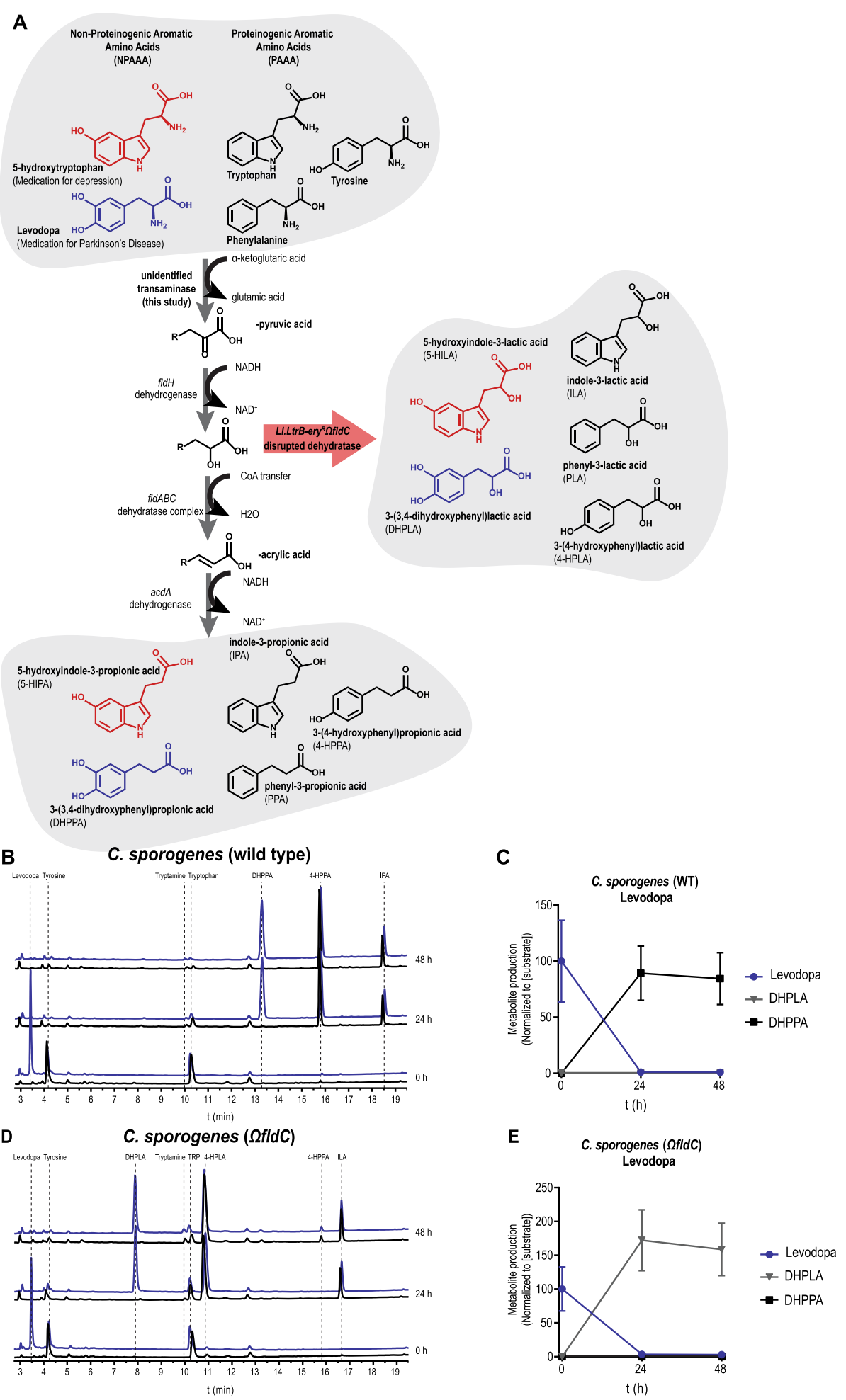

Fig. 1 (See legend on next page.) 
(See figure on previous page.)

Fig. 1 Levodopa is deaminated by Clostridium sporogenes. a Full reductive deamination pathway of C. sporogenes is depicted resulting in the full deamination (R-propionic acid) of (non)-proteinogenic aromatic amino acids ((N)PAAAs). The red arrow indicates a disrupted deamination pathway of $C$. sporogenes, where the dehydratase subunit fldC is mutagenized, resulting in a pool of partially deaminated metabolites (R-lactic acid) by $C$. sporogenes. $\mathbf{b}$ HPLC-ED curves from supernatant of a C. sporogenes batch culture conversion of levodopa (3-(3,4-dihydroxyphenyl)alanine) over time. At the beginning of growth (timepoint $0 \mathrm{~h}$ ), $100 \mu \mathrm{M}$ of levodopa (blue) is added to the culture medium; the black line in the chromatogram depicts the control samples. In $24 \mathrm{~h}$, levodopa is completely converted to DHPPA (3-(3,4-dihydroxyphenyl)propionic acid), the deaminated product of levodopa. Other aromatic amino acids from the medium, tryptophan and tyrosine (which are detectable with ED), are converted to the deaminated products IPA (indole-3propionic acid) and 4-HPPA (3-(4-hydroxyphenyl)propionic acid). c Quantification $(n=3)$ of levodopa conversion to DHPPA by C. sporogenes wild type (also see Additional File 1: Table S1). $\mathbf{d}$ Analysis of the supernatant of CS ${ }^{\Omega f l d C}$ shows that levodopa is not deaminated to DHPPA but to its intermediate product DHPLA (3-(3,4-dihydroxyphenyl)lactic acid) within $24 \mathrm{~h}$. Tryptophan and tyrosine are converted to their intermediates ILA (indole-3-lactic acid) and 4-HPLA (3-(4-hydroxyphenyl)lactic acid), respectively. e Quantification $(n=3)$ of levodopa conversion to DHPLA by C. sporogenes $\Omega$ fldC (also see Additional File 1: Table S1). All experiments were performed in 3 independent biological replicates, and means with error bars representing the SEM are depicted

by the dehydrogenases (FldH and AcdA) and dehydratase (FldABC) (Fig. 1a). However, the gene encoding this transaminase remains unidentified. To further investigate this critical step in the pathway, all nine class I/II aminotransferases encoded by $C$. sporogenes were cloned, purified, and screened for their activity on levodopa and the other (N)PAAAs. Screening revealed a single aminotransferase (EDU38870 encoded by CLOSPO_01732) to be involved in their transamination (Fig. 2a). To verify whether other aminotransferases could substitute for the identified aminotransferase in vivo, CLOSPO_01732 was disrupted (resulting in $\mathrm{CS}^{\Omega C L O S P O} 01732$ (Additional File 1: Fig. S3A)) and a targeted metabolomic analysis of all the (N)PAAA metabolites was performed using HPLC-ED (except metabolites from phenylalanine, which were quantified using HPLCUV). The disruption of fldC or CLOSPO_01732 resulted in only a minor reduction of the exponential growth rate in rich broth (doubling time is $55.1 \pm 1.2 \mathrm{~min}$ and $64.1 \pm$ $1.1 \mathrm{~min}$, respectively, compared to wild type $44.3 \pm 1.2$ min) all reaching stationary phase within $12 \mathrm{~h}$ (Additional File 1: Fig. S3B). Comparing the metabolic profiles from wild type C. sporogenes $\left(\mathrm{CS}^{\mathrm{WT}}\right), \mathrm{CS}^{\Omega f l d C}$, and CS $S^{\Omega C L O S P O} 01732$ demonstrated that none of the other tested aminotransferases could take over this transaminase reaction effectively, except for the substrate phenylalanine (Fig. 2b and Additional File 1: Table S1). Disrupting CLOSPO_01732 significantly reduced the production of phenyl-3-propionic acid (PPA), 3-(4hydroxyphenyl)propionic acid (4-HPPA), indole-3propionic acid (IPA), and 3-(3,4-dihydroxyphenyl)propionic acid (DHPPA) by $16.4 \%, 79.0 \%, 97.2 \%$, and $97.7 \%$, respectively, compared to $\mathrm{CS}^{\mathrm{WT}}$ within $24-48 \mathrm{~h}$ (Additional File 1: Table S1). Presumably, the transamination of phenylalanine is substituted by EDU37030 as this aminotransferase also showed phenylalanine-converting activity in vitro (Fig. 2a). Interestingly, $\mathrm{CS}^{\Omega C L O S P O} 01732$ produces significantly higher amounts of tryptamine (4to 6 -fold increase at 24 and $48 \mathrm{~h}$, respectively) compared to $\mathrm{CS}^{\mathrm{WT}}$, reflecting a reduced competition for the same substrate by different enzymes (Fig. 2b, Additional File 1: Table S1). Analogous to tryptamine, $\mathrm{CS}^{\Omega C L O S P O \_01732}$ produced significantly more serotonin compared to $\mathrm{CS}^{\mathrm{WT}}$ at $48 \mathrm{~h}$ when incubated with 5-HTP (Fig. 2b, Additional File 1: Fig. S3C, Additional File 1: Table S1), though to a much lesser extent ( $1 \%$ of substrate added) compared to tryptamine. Collectively, the data show that the aromatic aminotransferase (EDU38870) is involved in the initiation of the aromatic amino acid deamination pathway and is crucial for the production of DHPPA, 5HILA, 5-HIPA, and the previously described metabolites to be circulating in the blood, IPA, and 4-HPPA (Dodd et al. [11]).

\section{3-(3,4-Dihydroxyphenyl)propionic acid elicits an inhibitory effect on ileal muscle contractions ex vivo}

Because levodopa is the main treatment of PD patients and is efficiently deaminated to DHPPA within $24 \mathrm{~h}$ by the $C$. sporogenes deamination pathway compared to 5HTP, we further focused on levodopa and its deamination products. DHPPA is a phenolic acid (a molecule in the class polyphenols), and recent findings demonstrated an association between bacterial-derived polyphenol metabolites and gut transit times in humans [27]. Levodopa is mainly absorbed in the proximal small intestine, but significant amounts can reach the distal part of the intestinal tract [17], and these levels increase with age [28]. As levodopa is taken orally, the first intestinal site where anaerobic bacteria such as C. sporogenes (Clostridium Cluster I) can encounter relevant levels of levodopa is the ileum. Studies on asymptomatic ileostomy subjects established that the core ileal microbiota consists of (facultative) anaerobes including species from Clostridium Cluster I [29, 30]. Moreover, the transit time in the SI has been shown to be significantly longer in PD patients compared to healthy controls (with a median increase of $1.75 \mathrm{~h}$ in PD patients) $[23,24]$. To this end, we tested whether DHPPA $(100 \mu \mathrm{M})$ could affect the muscle contractility in the ileum. Ileal rings of wild type C57BL/6 J mice were suspended in an ex vivo organ bath system to 
A

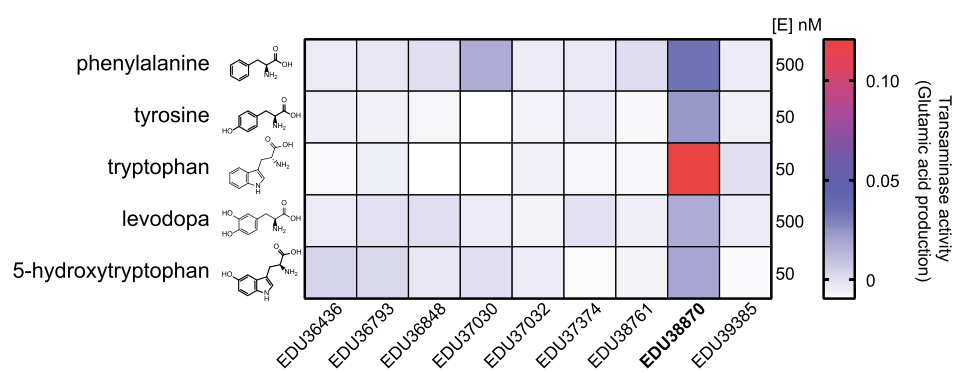

B

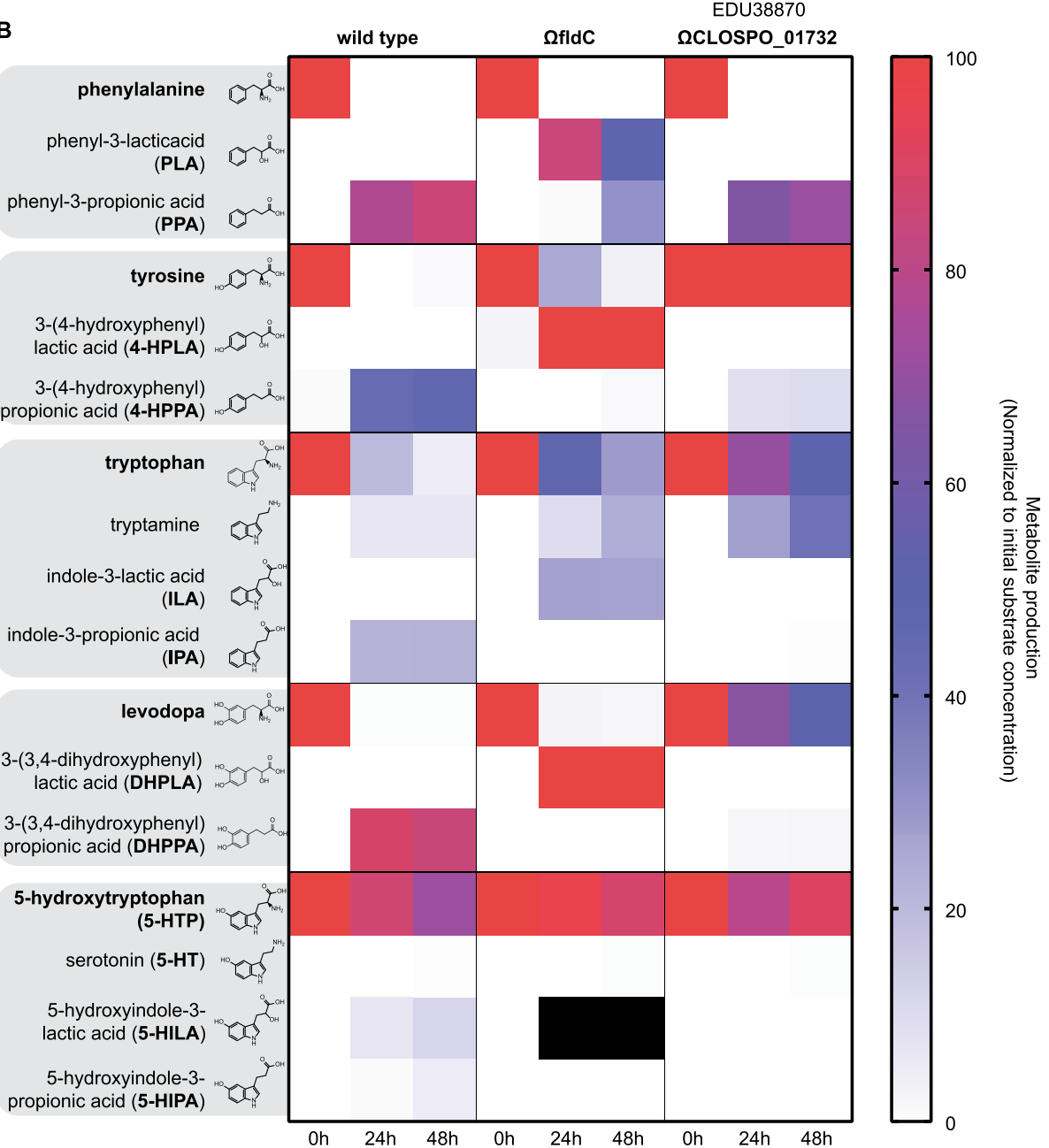

Fig. 2 Identification of the aromatic amino transferase initiating the deamination pathway. In order to identify which aminotransferase is responsible for the initial transaminase reaction, all class I/II aminotransferases were cloned and purified to test the activity against (N)PAAAs. a Transaminase activity (production of glutamate) for all substrates is depicted. EDU38870 (CLOSPO_01732) was involved in all transaminase reactions. EDU37030 showed similar activity as EDU38870, for phenylalanine. Experiment was performed in technical duplicates to screen for candidate genes for mutagenesis in $C$. sporogenes. b Targeted metabolic quantification of deamination products from $\mathrm{CS}^{\mathrm{WT}}, \mathrm{CS}^{\Omega \text { ffld C }}$, and CS ${ }^{\Omega \mathrm{CLOSPO}} 01732$ reveals that EDU38870 is involved in the transamination of all for all tested (N)PAAAs. All quantified deamination products are normalized to their initial substrate concentration, and the data represents 3 independent biological replicates. Corresponding values are reported, and metabolite concentration differences between WT and $\Omega f l d C$ or

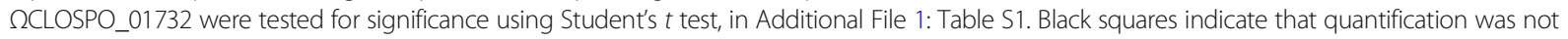
possible because of a coeluting HPLC-ED peak. As no commercial standards are available for 5-HILA and 5-HIPA, the peaks were quantified assuming a similar ED-detector response as for 5-HTP 
test the effect of DHPPA on muscle contractions. Our initial results indicated that DHPPA displayed an inhibiting effect on natural ileal contractility (Additional File 1: Fig. S4A).

Because acetylcholine is the neurotransmitter constantly produced from the excitatory muscle motor neurons to induce gut smooth muscle contractility [31], we tested whether DHPPA could have an inhibiting effect on acetylcholine-induced contractility in the ileum. The differences in amplitude of the contractions were quantified by measuring the decrease of the observed frequencies after a Fourier transform of 5-min intervals (Fig. 3a). Ileal tissue preparations were tested by initiating an acetylcholinergic twitch by adding $50 \mu \mathrm{M}$ of acetylcholine (a concentration saturating the muscarinic receptors $(\mathrm{Kd}=1.7 \pm 0.18 \mu \mathrm{M}$ [32] $)$. After $5 \mathrm{~min}$, $100 \mu \mathrm{M}$ DHPPA (a concentration resembling the higher levels detected in fecal samples of PD patients, see

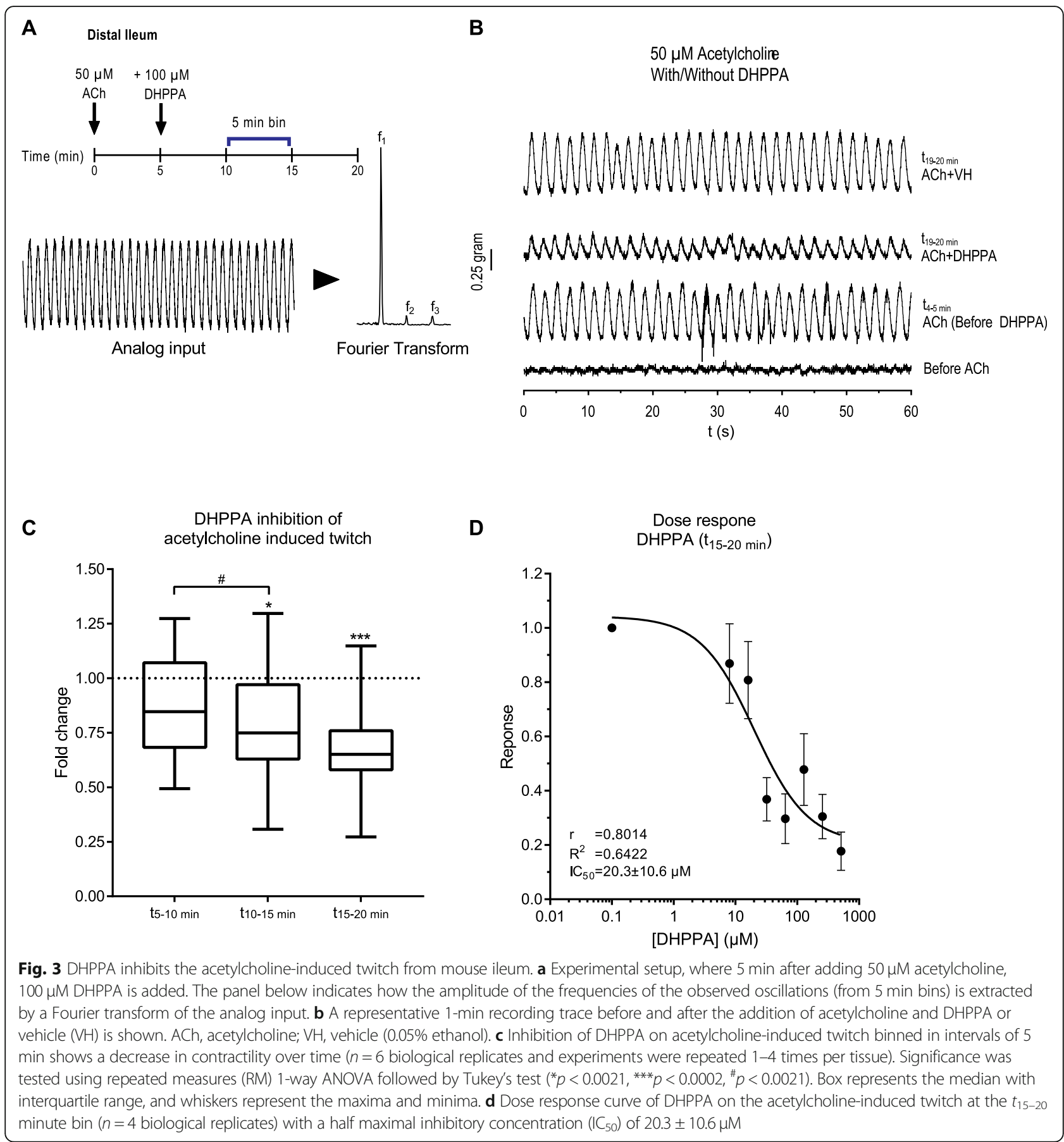


below) was added and contractions were followed further over a period of $15 \mathrm{~min}$. One-minute traces of the contractility representing one of the experiments are shown before and after addition of acetylcholine, DHPPA, or vehicle (Fig. 3b). A significant decrease in the amplitude (binned in 5-min intervals) of the acetylcholinergic twitch by DHPPA was observed at the 10-15-min (maximal reduction 69\%) and 15-20-min interval (maximal reduction 73\%) (Fig. 3c). In order to determine the potency of DHPPA, a dose response curve with DHPPA was performed and showed a half maximal inhibitory concentration $\left(\mathrm{IC}_{50}\right)$ of $20.3 \pm$ $10.6 \mu \mathrm{M}$ (Fig. 3d). In contrast to DHPPA, incubations with levodopa did not show any significant effect on the acetylcholinergic twitch (Additional File 1: Fig. S4B). Collectively, the data shows that DHPPA can inhibit the acetylcholine-induced muscle contractility of mouse ileum ex vivo.

\section{Active levodopa deamination pathway in fecal suspensions of patients with Parkinson's disease}

We hypothesized that if C. sporogenes or other bacteria with the deamination pathway (C. botulinum, Peptostreptococcus anaerobius, or Clostridium cadaveris [11]) are present in the GI tract of PD patients on levodopa/carbidopa treatment, those patients might have considerable amounts of DHPPA in their distal GI tract. Because DHPPA can be a product of gut bacterial metabolism of polyphenolic rich foods in the colon such as coffee and fruit (Jenner et al. [33]), fecal samples from 10 PD patients were compared to 10 age-matched HC. Samples were collected in a previous study, and there were no significant differences in macronutrients, dietary fiber, or total calorie intake between groups [34]. Using a catechol extraction targeted for the quantification of DHPPA, we found that the DHPPA concentrations were significantly higher in $\mathrm{PD}$ patient's fecal samples compared to $\mathrm{HC}$ (Fig. 4a). Identification of DHPPA was confirmed by LC-MS (Additional File 1: Table S2). The higher amounts (2.2-fold increase) of DHPPA observed in the fecal samples of PD patients are likely to result from levodopa metabolized by the anaerobic bacteria, deaminating levodopa through the FldBC dehydratase (Fig. 1a). In order to investigate the presence and activity of the anaerobic deamination pathway in fecal samples, the dehydration of the intermediate levodopa metabolite, DHPLA (Fig. 1a), was tested. The levodopa intermediate DHPLA was used as substrate instead of levodopa to prevent an in vitro substrate bias for bacteria that can decarboxylate levodopa to dopamine $[14,15]$. Moreover, FldABC is the key protein complex responsible for the production of DHPPA. Screening for the identified transaminase or FldH dehydrogenase upstream of FldABC would not be relevant as many bacterial species harbor these types of enzymes (Additional File 1: Fig. S5). Hence, fecal suspensions $(10 \% \mathrm{w} / \mathrm{v})$ from $\mathrm{PD}$ and $\mathrm{HC}$ were incubated anaerobically with DHPLA, and samples were collected at 0,20 , and $45 \mathrm{~h}$ and were analyzed by HPLC-ED. After $20 \mathrm{~h}$, DHPPA was detected in fecal samples from PD patients, as well as in fecal samples of $\mathrm{HC}$ when supplied with the substrate levodopa (Fig. 4b, Additional File 1: Fig. S6A). Moreover, DHPPA was further converted to the downstream dehydroxylated metabolite of DHPPA, 3-(3-hydroxyphenyl)propionic acid (3-HPPA), over time (Fig. 4b, Additional File 1: Fig. S6A). Because DHPPA is further converted to 3-HPPA in vitro, we quantified both the production of DHPPA and/or 3-HPPA in the fecal incubations as measure for the presence of an active deamination pathway. Metabolic profiles of PD or HC samples that produced DHPPA/3-HPPA over time were quantified and merged (Fig. 4c, Additional File 1: Fig. S6B), showing that DHPPA is produced first and is further metabolized to 3-HPPA. The production of DHPPA or 3-HPPA was observed in $50 \%$ and $20 \%$ of the PD patient's and $\mathrm{HC}$ fecal suspensions, respectively, after $20 \mathrm{~h}$ and in $70 \%$ and $50 \%$ PD patient's and HC fecal suspensions, respectively, after $45 \mathrm{~h}$ (Additional File 1: Fig. S6C). The production of 3-HPPA in vitro is likely to be performed by Eggerthella lenta, which has been shown to perform $p$-dehydroxylations [35]. Indeed, in vitro culturing of E. lenta showed $p$-dehydroxylation of DHPPA (Additional File 1: Fig. S7A [35, 36]). Because DHPPA is further converted to 3-HPPA in vitro, we examined whether 3-HPPA could elicit a similar effect on the acetylcholine-induced contractions in the ileum. Unlike, DHPPA, 3-HPPA did not elicit a significant effect on the acetylcholine-induced twitch (Additional File 1: Fig. S7B $[35,36])$. Furthermore, to investigate the genomic abundance levels of bacteria capable of deaminating (N)PAAAs, we analyzed the $16 \mathrm{~s}$ rDNA sequence data of the fecal samples of patients with Parkinson's disease [34] that were employed in this study (Additional File 1: Supplementary Results [1113, 37] and Additional File 1: Fig. S8). A significant positive correlation $\left(r=0.62, R^{2}=0.38, p=0.02\right)$ was found between bacteria with the deamination pathway and DHPPA/3HPPA production in fecal incubation samples at $20 \mathrm{~h}$ (Additional File 1: Fig. S8E). Taken together, the results show that DHPPA can be produced by the microbiota via anaerobic deamination of levodopa. Moreover, our findings indicate that 3HPPA originates from DHPPA via dehydroxylation potentially by Eggerthella lenta and that the aromatic deamination pathway, as measured by the production 


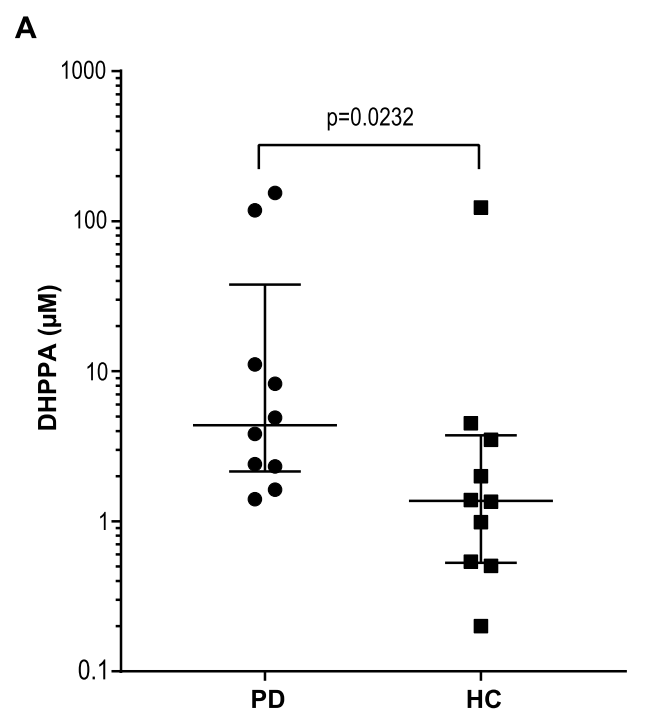

B

PD7
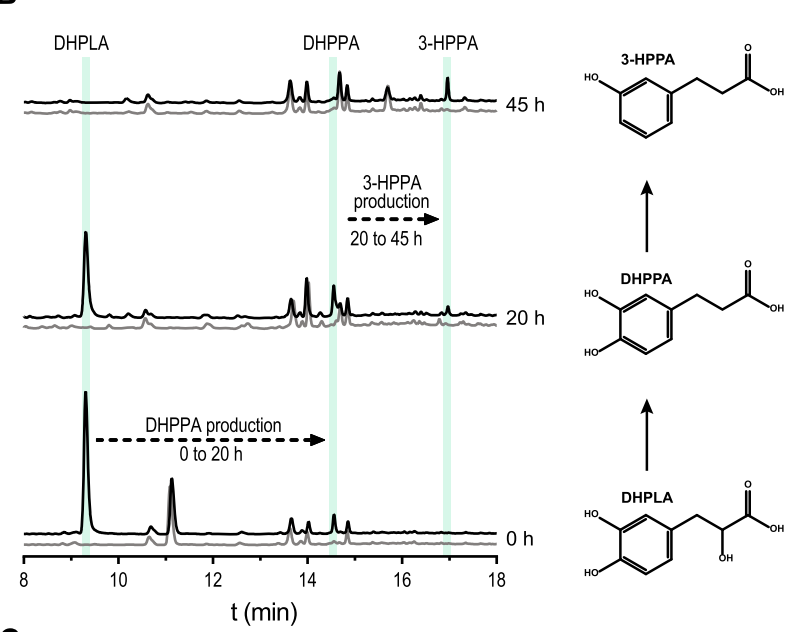

C
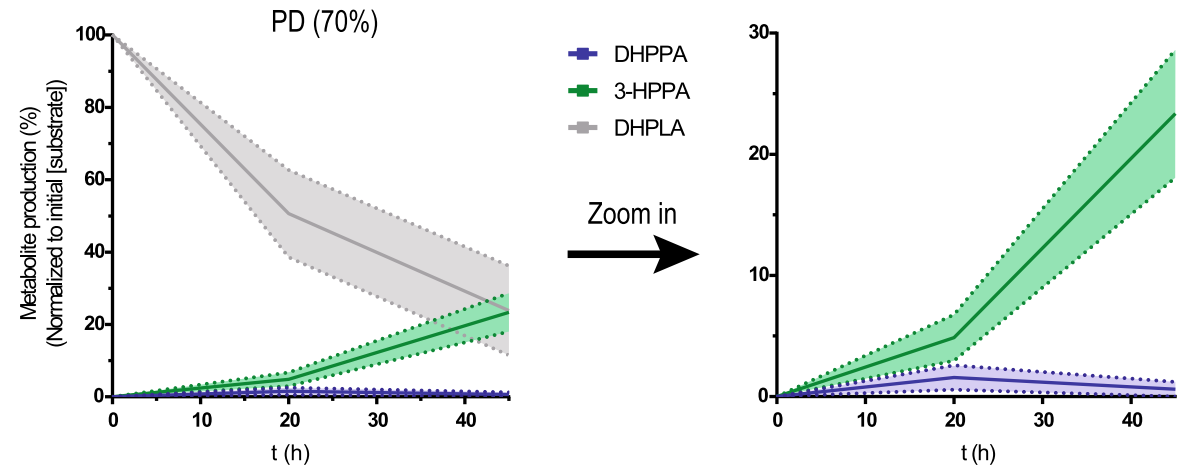

Fig. 4 (See legend on next page.) 


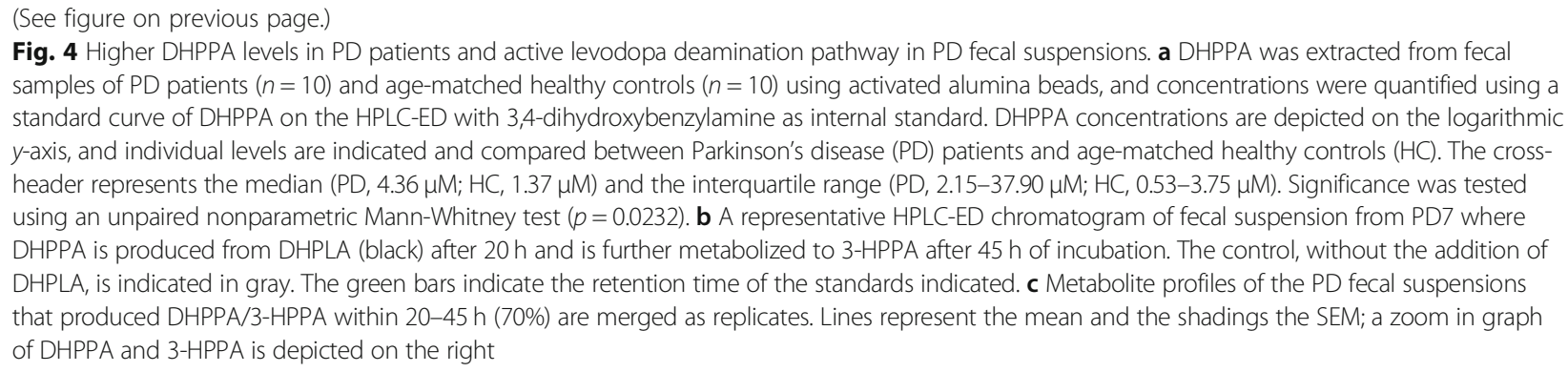

of DHPPA or 3-HPPA, is active and present in at least $70 \%$ of the PD samples.

\section{Discussion}

Identifying bacterial pathways and elucidating their potential impact on bacterial drug metabolism are crucial in order, not only, to maximize medication efficacy, but also to recognize and eventually prevent potential side effects that might affect the host's physiology on an individual basis [14, 36, 38, 39]. Here, we disclosed the reductive anaerobic deamination pathway in C. sporogenes by identifying its initiating enzyme, the aromatic aminotransferase, and expanded the pathway's relevance by demonstrating its capacity to convert two clinically important NPAAAs, levodopa and 5-HTP. We showed that C. sporogenes is able to completely deaminate levodopa to DHPPA and to a much lesser extent 5-HTP (Figs. 1 and 2; Additional File 1: Fig. S1, S2, S3; Additional File 1: Table S1). Disrupting the bacterial transaminaseencoding gene abolished the production of deaminated products, and increased the production of neuromodulators such as tryptamine and, to much lesser extent, serotonin (Fig. 2, Additional File 1: Fig. S3C, and Additional File 1: Table S1). Tryptamine is a natural product produced by $C$. sporogenes that has been proposed to modulate gut transit time [40]. The application of engineered gut bacteria as a therapeutic strategy to modulate GI motility or host physiology has been also proposed recently in two proof-of-concept studies: by heterologous expression of Ruminococcus gnavus tryptophan decarboxylase in Bacteroides thetaiotamicron [5] and by modification of the metabolic output of bioactive compounds in an engineered $f l d C$-deficient $C$. sporogenes strain [11]. However, translation of these studies into applications is hindered by restrictions on the application of genetically engineered microorganisms (GMOs) per se, and the complexity of introducing these GMOs into an existing gut microbiota ecosystem. Selective therapeutic blockage of the aminotransferase identified in this study may provide an attractive alternative solution to modify gut microbiota metabolism.

PD patients encounter increased gut transit time; thus, an additional inhibition of acetylcholine-induced contraction could result in further slowing down of gut transit rates. The inhibitory effect of DHPPA on the acetylcholine-induced ileal muscle contractions (Fig. 3), higher DHPPA levels in fecal samples of PD patients compared to $\mathrm{HC}$ (Fig. 4), and an active deamination pathway of levodopa during fecal incubations of PD patients (Fig. 4) demonstrate active deamination of levodopa in the distal GI tract of PD patients and suggest potential side effects of this bacterial by-product of the unabsorbed residue of the medication. DHPPA shares similarity with dopamine structure except of the terminal amine group, which is substituted by a carboxyl group in DHPPA. Dopamine and dopamine agonists have been shown to inhibit methacholine (analog of acetylcholine) induced contraction, which is not mediated via dopamine receptors, in guinea pig jejunum in similar concentration ranges to DHPPA $\left(\mathrm{EC}_{50}\right.$ relaxation by dopamine $\sim 290 \mu \mathrm{M}$ ) [41], indicating that DHPPA might act on a similar mechanism. Collectively, although further research is needed to unravel the underlying mechanism, our results show that DHPPA inhibits the acetylcholine-induced muscle contractions in the ileum with implications on intestinal motility, often observed in PD patients.

\section{Conclusions}

The present study shows that $C$. sporogenes can effectively deaminate unabsorbed residues of levodopa in fecal samples from PD patients to 3-(3,4-dihydroxyphenyl)propionic acid, which reduces ileal motility ex vivo. Overall, our results highlight the urgency to unravel potential effects of gut bacterial processing of (unabsorbed residues of) medication, such as levodopa.

\section{Methods \\ Growth and incubation of Clostridium sporogenes and Eggerthella lenta}

Clostridium sporogenes ATCC15579 was grown in enriched beef broth (EBB) with $2 \mathrm{~g} / \mathrm{L}$ glucose [14] and $0.1 \%$ Tween $80(\mathrm{EBB} / \mathrm{T})$ anaerobically $\left(10 \% \mathrm{H}_{2}, 10 \%\right.$ $\mathrm{CO}_{2}, 80 \% \mathrm{~N}_{2}$ ) in a Don Whitley Scientific DG250 Workstation (LA Biosystems, Waalwijk, The Netherlands) at $37^{\circ} \mathrm{C}$. Eggerthella lenta DSM2243 was grown in 
modified DSMZ medium 78 (DSMZ 78: beef extract, $10.0 \mathrm{~g} / \mathrm{L} ; \quad$ casitone, $\quad 30.0 \mathrm{~g} / \mathrm{L}$; yeast extract, $5.0 \mathrm{~g} / \mathrm{L}$; $\mathrm{K}_{2} \mathrm{HPO}_{4}, 5.0 \mathrm{~g} / \mathrm{L}$; Tween 80, 0.1\%; menadione (vitamin $\mathrm{K} 3), 1 \mu \mathrm{g} / \mathrm{mL}$; cysteine, $0.5 \mathrm{~g} / \mathrm{L}$; hemin, $5 \mathrm{mg} / \mathrm{L}$; L-arginine $0.1-1.5 \%)$ anaerobically $\left(1.5 \% \mathrm{H}_{2}, 5 \% \mathrm{CO}_{2}\right.$, balance with $\mathrm{N}_{2}$ ) in a Coy Laboratory Anaerobic Chamber (neoLab Migge $\mathrm{GmbH}$, Heidelberg, Germany) at $37^{\circ} \mathrm{C}$ in a tube shaker at 500 RPM. Upon use, bacteria were inoculated from $-80^{\circ} \mathrm{C}$ stored glycerol stocks in the appropriate media and grown for $18-24 \mathrm{~h}$ for C. sporogenes and 24-40 h for E. lenta. Overnight turbid cultures were then diluted $1 / 50$ in an appropriate volume $\mathrm{EBB} / \mathrm{T}$ or CMM for further experiments with $100 \mu \mathrm{M}$ levodopa (D9628, Sigma), 5-hydroxytrytophan (H9772, Sigma), $50 \mu \mathrm{M}$ 3-(3,4-dihydroxyphenyl)propionic acid (102601, Sigma), or $\mathrm{H}_{2} \mathrm{O}$ as control. All experiments were performed in triplicate (3 biological replicates).

\section{Protein production and purification}

Transaminase-encoding genes from $C$. sporogenes (Additional File 1: Table S3) were amplified using Phusion High-fidelity DNA polymerase and primers listed in Additional File 1: Table S3. All amplified genes were cloned in pET15b, except for EDU37032 which was cloned in pET28b (Additional File 1: Table S3). Plasmids were maintained in E. coli $\mathrm{DH} 5 \alpha$ and verified by Sanger sequencing before transformation to E. coli BL21 (DE3). Overnight cultures were diluted 1:50 in fresh LB medium with the appropriate antibiotic and grown to OD600 $=0.7-0.8$ shaking at $37^{\circ} \mathrm{C}$. Protein translation was induced with $1 \mathrm{mM}$ isopropyl $\beta$-D-1-thiogalactopyranoside (IPTG, 1141144 6001, Roche Diagnostics), and cultures were incubated overnight at $18^{\circ} \mathrm{C}$. The cells were washed with $1 / 5$ th of the volume in $1 \times$ ice-cold PBS and stored at $-80^{\circ} \mathrm{C}$ or directly used for protein isolation. Cell pellets were thawed on ice and resuspended in 1/50th of buffer A $(300 \mathrm{mM} \mathrm{NaCl} ; 10 \mathrm{mM}$ imidazole; $50 \mathrm{mM}$ KPO4, pH 8.0) containing $0.2 \mathrm{mg} / \mathrm{mL}$ lysozyme (105281, Merck) and $2 \mu \mathrm{g} / \mathrm{mL}$ DNAse (11284932001, Roche Diagnostics), and incubated for at least $10 \mathrm{~min}$ on ice before sonication ( 10 cycles of $15 \mathrm{~s}$ with $30 \mathrm{~s}$ cooling at $8 \mu \mathrm{m}$ amplitude) using Soniprep-150 plus (Beun de Ronde, Abcoude, The Netherlands). Cell debris was removed by centrifugation at $20,000 \times g$ for $20 \mathrm{~min}$ at $4{ }^{\circ} \mathrm{C}$. The $6 \times$ his-tagged proteins were purified using a nickel-nitrilotriacetic acid (Ni-NTA) agarose matrix (30250, Qiagen). Cell-free extracts were loaded on $0.5 \mathrm{~mL} \mathrm{Ni-NTA}$ matrixes and incubated on a roller shaker for $2 \mathrm{~h}$ at $4{ }^{\circ} \mathrm{C}$. The Ni-NTA matrix was washed three times with $1.5 \mathrm{~mL}$ buffer $\mathrm{B}$ $(300 \mathrm{mM} \mathrm{NaCl} ; 20 \mathrm{mM}$ imidazole; $50 \mathrm{mM} \mathrm{KPO} 4, \mathrm{pH}$ 8.0) before elution with buffer $\mathrm{C}(300 \mathrm{mM} \mathrm{NaCl} ; 250$ $\mathrm{mM}$ imidazole; $50 \mathrm{mM}$ KPO4, $\mathrm{pH} 8.0$ ). Imidazole was removed from purified protein fractions using Amicon Ultra centrifugal filters (UFC505024, Merck) and washed three times and reconstituted in buffer D (50 $\mathrm{mM}$ Tris- $\mathrm{HCl} ; 300 \mathrm{mM} \mathrm{NaCl} ; \mathrm{pH}$ 7.5). Protein concentrations were measured spectrophotometrically (Nanodrop 2000, Isogen, De Meern, The Netherlands) using the predicted extinction coefficient and molecular weight from ExPASy ProtParam tool (www.web. expasy.org/protparam/).

\section{Transaminase activity test}

Purified transaminases were incubated with $1 \mathrm{mM}$ substrate, $2 \mathrm{mM} \alpha$-ketoglutaric acid, and $0.1 \mathrm{mM}$ PLP (pyridoxal-5-phosphate, P9255, Sigma, The Netherlands) in buffer D with an enzyme concentration of $50 \mathrm{nM}$ for tyrosine, tryptophan, or 5-HTP as substrate and an enzyme concentration of $500 \mathrm{nM}$ for phenylalanine and levodopa as substrate. Enzyme reactions were incubated for $0.5 \mathrm{~h}$ at $37^{\circ} \mathrm{C}$; the reactions were stopped with $0.7 \%$ (v/v) perchloric acid (1:1). Transaminase activity was tested using an L-glutamic acid detection kit (K-GLUT, Megazyme Inc., Wicklow, Ireland), according to the manufacturer's microplate assay procedure with some modifications. The supplied buffer was substituted for buffer D (described above, to prevent oxidation of the substrates/products). A reaction mix was prepared mixing $50 \mu \mathrm{L}$ buffer $\mathrm{D}, 10 \mu \mathrm{L}$ quenched sample reaction mixture, $20 \mu \mathrm{L} \mathrm{NAD}$ /iodonitrotetrazolium chloride solution, $5 \mu \mathrm{L}$ diaphorase solution, and $5 \mu \mathrm{L}$ glutamate dehydrogenase (GIDH) solution and reconstituted to a final volume of $290 \mu \mathrm{L}$ with $\mathrm{H}_{2} \mathrm{O}$. Absorbance at $492 \mathrm{~nm}$ was measured after $10 \mathrm{~min}$ of incubation using a microplate reader (Synergy HTX spectrophotometer, BioTek, BioSPX, The Netherlands), and background was subtracted from initial read before addition of GIDH solution.

\section{Targeted mutagenesis}

Gene disruptions in Clostridum sporogenes were performed using the ClosTron system [42, 43]. This system facilitates targeted mutagenesis using the group II Ll.LtrB intron of Lactococcus lactis. Introns targeting fldC (CLOSPO_311) or CLOSPO_1732 (encoding for the transaminase) were designed using the ClosTron intron design tool (http://www.clostron.com) and were ordered in pMTL007C-E2 from ATUM (Newark, CA, USA) resulting in pMTL007C-E2_Cs-fldC-561a and pMT L007C-E2_Cs-CLOSPO_1732-493s, respectively. Plasmids were transferred to $C$. sporogenes by conjugation as described before [43] using E. coli CA434 (E. coli HB101 (Bio-Rad Laboratories, The Netherlands)) harboring the broad host IncPß ${ }^{+}$conjugational plasmid pRK24 [44] as donor strain. E. coli CA434 harboring pMTL007C-E2_CsfldC-561a or pMTL007C-E2_Cs-CLOSPO_1732-493s was 
grown in Luria Broth (LB) with $10 \mu \mathrm{g} / \mathrm{mL}$ tetracycline and $25 \mu \mathrm{g} / \mathrm{mL}$ chloramphenicol (to select for pRK24 and PMTL007C-E2, respectively). Cell suspensions of $1 \mathrm{~mL}$ of overnight culture were washed once with PBS, and the cell pellet was resuspended in $200 \mu \mathrm{L}$ of C. sporogenes overnight cell suspension. The bacterial mixture was spotted (in drops of $10 \mu \mathrm{L}$ ) on trypticase soy agar (TSA) plates and incubated for $24 \mathrm{~h}$ anaerobically at $37^{\circ} \mathrm{C}$. Sequentially, 1 $\mathrm{mL}$ of PBS was added to the spotted plates and the donorrecipient mix was scraped of the plate; sequentially, the scraped-off suspension was distributed over TSA plates containing $50 \mu \mathrm{g} / \mathrm{mL}$ neomycin (to prevent growth of $E$. coli) and $15 \mu \mathrm{g} / \mathrm{mL}$ chloramphenicol to select for C. sporogenes conjugants. Chloramphenicol-resistant colonies of C. sporogenes were re-streaked on TSA plates containing $50 \mu \mathrm{g} / \mathrm{mL}$ neomycin and $2.5 \mu \mathrm{g} / \mathrm{mL}$ erythromycin (to select for intron insertion) for several times. To make sure the plasmids were integrated, colonies were checked and selected for their sensitivity towards chloramphenicol and the genomic DNA was verified using PCR (Additional File 1: Fig. S1F and 2A).

\section{Fecal samples from patients with Parkinson's disease and age-matched healthy controls}

Fecal samples from patients diagnosed with PD $(n=10)$ and age-matched healthy controls $(n=10)$ were acquired from the Movement Disorder Center at Rush University Medical Center, Chicago, IL, USA, published previously [34]. All study subjects consented to the use of their samples for research. PD was diagnosed according to the UK Brain Bank Criteria as previously described [34]. Study subjects were provided with the supplies and instructions for home feces collection using the BD Gaspak EZ Anaerobe Gas Generating Pouch System with Indicator (Ref 260683; Becton, Dickinson and Company, Sparks, MD) in order to minimize the exposure of the feces to high oxygen ambient atmosphere, which may alter the microbiota. Subjects were asked to have a bowel movement within $24 \mathrm{~h}$ of their study visit. Subjects kept the sealed anaerobic fecal bag in a cold environment, before bringing the anaerobic fecal bag to the hospital. Fecal samples were then immediately stored at $-80^{\circ} \mathrm{C}$ until analysis.

\section{Fecal metabolite incubations from PD patients and HC subjects}

Stool samples were suspended 1:1 (w/v) in EBB/T and incubated anaerobically $\left(10 \% \mathrm{H}_{2}, 10 \% \mathrm{CO}_{2}, 80 \% \mathrm{~N}_{2}\right)$ in a Don Whitley Scientific DG250 Workstation (LA Biosystems, Waalwijk, The Netherlands) at $37^{\circ} \mathrm{C}$ with $100 \mu \mathrm{M}$ sodium 3(3,4-dihydroxyphenyl)-DL-lactate (39363, Sigma). Samples were taken at 0,20 , and $45 \mathrm{~h}$ and analyzed on HPLC-ED as described below.

\section{HPLC-ED/UV analysis and sample preparation}

For bacterial cell suspensions, $1 \mathrm{~mL}$ of methanol was added to $0.25 \mathrm{~mL}$ of cell suspension and stored at $-20{ }^{\circ} \mathrm{C}$ until further use. For fecal metabolite incubations, $300 \mu \mathrm{L}$ of methanol was added to $75 \mu \mathrm{L}$ of fecal suspension and stored at $-20{ }^{\circ} \mathrm{C}$ until further use. Metabolites from stool samples were extracted by suspending the stool $1: 1(\mathrm{w} / \mathrm{v})$ in water, followed by homogenization by vigorously vortexing while keeping samples as cold as possible. Homogenized suspensions were centrifuged at $3500 \times g$ for $20 \mathrm{~min}$ at $4{ }^{\circ} \mathrm{C}$, and sequentially, $1.6 \mathrm{~mL}$ of methanol was added to $0.4 \mathrm{~mL}$ of supernatant. From bacterial, fecal incubation or stool samples, cells and protein precipitates were removed by centrifugation at $20,000 \times g$ for $10 \mathrm{~min}$ at $4{ }^{\circ} \mathrm{C}$. Supernatant was transferred to a new tube, and the methanol fraction was evaporated in a Savant speed-vacuum dryer (SPD131, Fisher Scientific, Landsmeer, The Netherlands) at $60{ }^{\circ} \mathrm{C}$ for $1.5-2 \mathrm{~h}$. The aqueous fraction was reconstituted with $0.7 \% \mathrm{HClO}_{4}$ to the appropriate volume. Samples were filtered and injected into the HPLC-ED system (Alliance Separations Module 2695, Waters Chromatography B.V, Etten-Leur, The Netherlands; Dionex ED40 electrochemical detector, Dionex, Sunnyvale, USA, with a glassy carbon working electrode (DC amperometry at 0.8 or $1.0 \mathrm{~V}$, with $\mathrm{Ag} / \mathrm{AgCl}$ as reference electrode)). Samples were analyzed on a C18 column (Kinetex $5 \mu \mathrm{M}$, C18 $100 \AA, 250 \times 4.6 \mathrm{~mm}$, Phenomenex, Utrecht, The Netherlands) using a gradient of water/methanol with $0.1 \%$ formic acid $\left(0-10 \mathrm{~min}, 95-80 \% \mathrm{H}_{2} \mathrm{O} ; 10-20 \mathrm{~min}\right.$, 80-5\% $\mathrm{H}_{2} \mathrm{O} ; 20-23 \mathrm{~min}, 5 \% \mathrm{H}_{2} \mathrm{O} ; 23-31 \mathrm{~min}, 95 \%$ $\mathrm{H}_{2} \mathrm{O}$ ). Fecal suspension metabolites were injected twice and analyzed at DC amperometry at $0.8 \mathrm{~V}$ (for DHPPA) and at $1.0 \mathrm{~V}$ (for 3-HPPA). Lowering the voltage makes the detection more selective for more readily oxidizable compounds [45] such as DHPPA, but making 3-HPPA invisible for detection. For the detection of the C. sporogenes metabolites and for peak isolation, another HPLC-ED system was used (Jasco AS2059 plus autosampler, Jasco Benelux, Utrecht, The Netherlands; Knauer K-1001 pump, Separations, H. I. Ambacht, The Netherlands) with the same detector (ED40) and the same gradient as described above. Phenylalanine metabolites were detected by injecting the same samples in an HPLC-UV system (Alliance Separations Module 2695, Waters Chromatography B.V, Etten-Leur, The Netherlands; TSP UV6000LP UV-detector (wavelength, 260 nM) Thermo Scientific, The Netherlands). Samples for peak isolation were separated on a Vydac Semipreparative C18 column (218TP510, $5 \mu \mathrm{m}, 300 \AA$, 10 $\mathrm{mm} \times 250 \mathrm{~mm}$, VWR International B.V, Amsterdam, The Netherlands) at $3 \mathrm{~mL} / \mathrm{min}$ using the same gradient as above. Data recording and analysis were performed using Chromeleon software (version 6.8 SR13). 
Significance was tested using a two-sample equal variance (homoscedastic) Student's $t$ test (Microsoft Excel 2019 version 1808).

\section{Catechol extraction from stool for DHPPA quantification}

Catechols were extracted from PD patients and HC stool samples using activated alumina powder (199966, Sigma) as previously described [14] with a few modifications. A volume of $200 \mu \mathrm{L} 50 \%$ stool suspension (described above) was used with $1 \mathrm{mM}$ DHBA (3,4-dihydroxybenzylamine hydrobromide, 858781, Sigma) as an internal standard. Samples were adjusted to $\mathrm{pH} 8.6$ with $800 \mu \mathrm{L}$ TE buffer (2.5\% EDTA; $1.5 \mathrm{M}$ Tris/ $\mathrm{HCl}, \mathrm{pH} 8.6$ ), and 5$10 \mathrm{mg}$ of alumina was added. Suspensions were mixed on a roller shaker at room temperature for $20 \mathrm{~min}$ and were sequentially centrifuged for $30 \mathrm{~s}$ at $20,000 \times \mathrm{g}$ and washed three times with $1 \mathrm{~mL}$ of $\mathrm{H}_{2} \mathrm{O}$ by aspiration. Catechols were eluted using $0.7 \% \mathrm{HClO}_{4}$ and filtered before injection into the HPLC-ED-system as described above (DC amperometry at $0.8 \mathrm{~V}$ ). A standard curve was injected to quantify the concentrations of DHPPA in $50 \%(w / v)$ stool samples. Significance was tested using an unpaired nonparametric Mann-Whitney test (GraphPad Prism version 7).

\section{Organ-bath experiments}

Distal ileal samples were harvested from wild type adult (18-20 weeks) male C57BL/6 J mice that were sacrificed for another purpose. Harvested tissue was immediately removed, placed, and washed in $0.85 \%$ $\mathrm{NaCl}$. Approximately 3-mm rings were cut and were placed in an organ bath (Tissue Bath Station with SSL63L force transducer, Biopac Systems Inc., Varna, Bulgaria) filled with Krebs-Henseleit solution $(\mathrm{NaCl}$, $7.02 \mathrm{~g} / \mathrm{L} ; \quad \mathrm{KCl}, \quad 0.44 \mathrm{~g} / \mathrm{L} ; \quad \mathrm{CaCl}_{2} .2 \mathrm{H}_{2} \mathrm{O}, \quad 0.37 \mathrm{~g} / \mathrm{L}$; $\mathrm{MgCl}_{2} .6 \mathrm{H}_{2} \mathrm{O}, 0.25 \mathrm{~g} / \mathrm{L} ; \mathrm{NaH}_{2} \mathrm{PO}_{4} . \mathrm{H}_{2} \mathrm{O}, 0.17 \mathrm{~g} / \mathrm{L} ;$ glucose, $\left.2.06 \mathrm{~g} / \mathrm{L} ; \mathrm{NaHCO}_{3}, 2.12 \mathrm{~g} / \mathrm{L}\right)$ gassed with carbogen gas mixture $\left(5 \% \mathrm{CO}_{2}\right.$, balanced with $\left.\mathrm{O}_{2}\right)$ at $37^{\circ} \mathrm{C}$. Ileal rings were equilibrated for at least 45-60 min with replacement of Krebs-Henseleit solution approximately every $15 \mathrm{~min}$. Sequentially, $50 \mu \mathrm{M}$ of acetylcholine (ACh) (Sigma, A2661) was added to induce a stable repetitive muscle twitch response, and after $\sim 5$ min, $100 \mu \mathrm{M}$ of DHPPA (102601, Sigma) ( $n=6$ biological replicates, 1-4 technical replicates), 3-HPPA (91779, Sigma) ( $n=4$ biological replicates, 2 technical replicates), or levodopa (D9628, Sigma) ( $n=3$ biological replicates, 2 technical replicates) was added for $\sim 15$ min before the ileal rings were washed. This step was repeated 1-4 times per ileal preparation. As control, ACh was added for at least 20 min with or without $0.05 \%$ ethanol (solvent of DHPPA) after $5 \mathrm{~min}$ to check for spontaneous decrease. For the dose response curve ( $n=4$ biological replicates), every 15 min, the cumulative dose of DHPPA was increased by 2 -fold ranging from 8 to $512 \mu \mathrm{M}$. Data was recorded and analyzed in BioPac Student Lab 4.1 (Build: February 12, 2015). Frequencies were extracted performing a fast Fourier transform (FFT) on bins of 5-min intervals. The maximum amplitude of all the observed frequencies was extracted, and the average decrease of all frequencies over time was calculated. Significance was tested using repeated measures (RM) 1-way ANOVA followed by Tukey's test (GraphPad Prism version 7).

\section{NMR}

Samples were exchanged once with 99.9 atom\% $\mathrm{D}_{2} \mathrm{O}$ with intermediate lyophilization, finally dissolved in $650 \mu \mathrm{L} \mathrm{D} \mathrm{D}_{2} \mathrm{O}$. One- and two-dimensional ${ }^{1} \mathrm{H}$ and ${ }^{13} \mathrm{C}$ NMR spectra were recorded at a probe temperature of $25^{\circ} \mathrm{C}$ on a Varian Inova 500 spectrometer (NMR Department, University of Groningen). Chemical shifts are expressed in parts per million in reference to external acetone $\left(\delta{ }^{1} \mathrm{H} 2.225 ; \delta{ }^{13} \mathrm{C} 31.08\right)$. 1D $500-\mathrm{MHz} 1 \mathrm{H}$ NMR spectra were recorded with $5000 \mathrm{~Hz}$ spectral width at $16 \mathrm{k}$ complex data points, using a WET1D pulse to suppress the HOD signal. Homonuclear decoupled ${ }^{1} \mathrm{D}$ $125 \mathrm{MHz}{ }^{13} \mathrm{C}$ NMR spectra were recorded with 31,000 $\mathrm{Hz}$ spectral width at $64 \mathrm{k}$ complex data points. $2 \mathrm{D}$ ${ }^{1} \mathrm{H}_{-}{ }^{13} \mathrm{C}$ HSQC spectroscopy was performed using multiplicity editing, rendering $\mathrm{CH}_{2}$ signals in the negative plane, while $\mathrm{CH}$ and $\mathrm{CH}_{3}$ remain in the positive plain. 2D ${ }^{13} \mathrm{C}-{ }^{1} \mathrm{H}$ HMBC spectroscopy was performed suppressing single-bond correlations. Spectra were processed using MestReNova v9.1 (Mestrelabs Research SL, Santiago de Compostela, Spain).

\section{LC-MS}

HPLC-MS analysis was performed using an Accella1250 HPLC system coupled with the benchtop ESI-MS Orbitrap Exactive (Thermo Fisher Scientific, San Jose, CA, USA) in negative and positive ion mode. Samples were analyzed on a C18 column (Shim Pack Shimadzu XRODS $3 \times 75 \mathrm{~mm}$ ) using a gradient of water/acetonitrile with $0.1 \%$ formic acid $\left(0-5 \mathrm{~min}, 98-90 \% \mathrm{H}_{2} \mathrm{O} ; 5-10\right.$ min, 90-5\% $\mathrm{H}_{2} \mathrm{O} ; 10-13 \mathrm{~min}, 5 \% \mathrm{H}_{2} \mathrm{O} ; 13-14 \mathrm{~min}, 98 \%$ $\mathrm{H}_{2} \mathrm{O}$ ). Data analysis was performed using Qual Browser Thermo Xcalibur software (version 2.2 SP1.48).

HPLC-MS analysis of alumina extraction samples was performed using a Waters Acquity Class-I UPLC (Waters Chromatography B.V, Etten-Leur, The Netherlands) system coupled to a MaXis Plus Q-TOF (Bruker, Billerica, MA, USA) on negative ion mode with post-column addition of $3 \mu \mathrm{L} / \mathrm{min}$ ESI Tune Mix (G1969-85000; Agilent Technologies, Middelburg, The Netherlands) for mass calibration. Samples were analyzed on a C18 column (Shim Pack Shimadzu XR-ODS $3 \times 75 \mathrm{~mm}$ ) using a 
gradient of water/acetonitrile with $0.1 \%$ formic acid $(0-5$ min, 98-90\% $\mathrm{H}_{2} \mathrm{O} ; 5-10 \mathrm{~min}, 90-5 \% \mathrm{H}_{2} \mathrm{O} ; 10-13 \mathrm{~min}, 5 \%$ $\mathrm{H}_{2} \mathrm{O} ; 13-15$ min, 2\% $\mathrm{H}_{2} \mathrm{O} ; 15-17 \mathrm{~min}, 98 \% \mathrm{H}_{2} \mathrm{O}$ ). Data analysis was performed using Bruker Compass Data Analysis (version 4.2 SR1).

\section{Bioinformatics \\ Phylogenetic trees}

Proteins were BLASTed against a local BLAST database constructed from the protein sequences of the NIH Human Microbiome Project (HMP) Roadmap project (PRJNA43021) using BLAST 2.9.0+, NCBI. The top 100 BLASTp hits were aligned in the Constraint-based Multiple Alignment Tool (COBALT, NCBI) and converted to a distance tree using NCBI TreeView (Parameters: Fast Minimum Evolution; Max Seq Difference, 0.85; Distance, Grishin).

\section{Sequence data analysis}

The demultiplexed paired-end sequence data from stool and sigmoid colon samples of PD patients and healthy controls from Keshavarzian et al. [34] (bioproject PRJNA268515) were analyzed using Kraken2 (v2.0.9, April 7, 2020), a $k$-mer taxonomic classification system [46], using the standard Kraken2 database. To further estimate the species abundance, the Kraken2 output was analyzed with Bracken (Bayesian Reestimation of Abundance with KrakEN; v2.6.0, April 3, 2020) [47]. The number of mapped reads from bacteria with the fld-gene cluster [11] was extracted from the Bracken results, and the abundance was calculated relative to the total number of mapped bacterial reads.

\section{Supplementary information}

Supplementary information accompanies this paper at https://doi.org/10. 1186/s12915-020-00876-3.

Additional file 1: Supplementary Results - Metagenomic analysis of deaminating bacteria and $E$. lenta in PD and $\mathrm{HC}$ fecal and mucosal samples. Fig. S1 - NMR and MS confirmation of levodopa product, 3-(3,4-

dihydroxyphenyl)propionic acid. Fig. S2 - 5-HTP conversion by Clostridium sporogenes. Fig. S3 - Growth curves of $\mathrm{CS}^{\Omega \text { fld } \mathrm{C}}$ and $C S^{\Omega C L O S P O} 01732$, and 5-

HT production. Fig. S4 - Initial effect of DHPPA on natural ileal contractility and no effect of levodopa on acetylcholine induced twitch. Fig. S5 -

Phylogenetic tree of C. sporogenes FldH and EDU38870. Fig. S6 - Fecalincubations from healthy age-matched controls. Fig. S7 - 3-HPPA is produced by E. lenta. Fig. S8 - Analysis of 16s rDNA metagenomics data of deaminating bacteria and E. lenta in PD and $\mathrm{HC}$ fecal and mucosal samples.

Table S1 - Values and statistical results corresponding to Fig. 2B. Table S2 - MS confirms that DHPPA is extracted from PD and HC samples using alumina extraction method. Table $\mathbf{S 3}$ - Plasmids and primers used in this study.

Additional file 2. All data underlying the main and supplementary figures and tables of the manuscript.

\section{Acknowledgements}

We thank Mr. Walid Maho, Interfaculty Mass Spectrometry Center, University of Groningen, The Netherlands, for running and analyzing the samples on the LC-MS and Prof. Dr. Michiel Kleerebezem, Host-Microbe Interactomics
Group, Wageningen University, The Netherlands, for critical reading of our manuscript.

\section{Authors' contributions}

S.P.K. and S.E.A. conceived and designed the study. S.P.K., H.R.J., S.L.W., S.S.L, S.A.N., H.P., and A.K. performed the experiments, and S.P.K., S.S.L., H.P., and S.E.A. analyzed the data. S.P.K. and S.E.A. wrote the original manuscript that was reviewed by S.S.L., S.A.N., H.P., and A.K. Funding was acquired by S.E.A. All authors read and approved the final manuscript.

\section{Funding}

S.E.A is supported by a Rosalind Franklin Fellowship, co-funded by the European Union and University of Groningen, The Netherlands.

\section{Availability of data and materials}

All data generated or analyzed during this study are included in this published article and its supplementary information files. The metagenomic sequence data collected by Keshavarzian et al. [34] were deposited under bioproject number PRJNA268515.

Ethics approval and consent to participate

Samples from participants were used from Keshavarzian et al. [34] (bioproject PRJNA268515) [46], where all subjects consented to use of their samples for research.

Consent for publication

Not applicable

\section{Competing interests}

The authors declare no competing interests.

\section{Author details}

1Department of Molecular Immunology and Microbiology, Groningen Biomolecular Sciences and Biotechnology Institute (GBB), University of Groningen, Nijenborgh 7, 9747 AG Groningen, The Netherlands. ${ }^{2}$ Current Address: Department of Laboratory Medicine, Cluster Human Nutrition \& Health, University Medical Center Groningen (UMCG), Hanzeplein 1, 9713 GZ Groningen, The Netherlands. ${ }^{3}$ Department of Molecular Neurobiology, Groningen Institute for Evolutionary Life Sciences (GELIFES), University of Groningen, Nijenborgh 7, 9747 AG Groningen, The Netherlands. ${ }^{4}$ Interfaculty Mass Spectrometry Center, University of Groningen, Groningen, The Netherlands. ${ }^{5}$ Division of Digestive Disease and Nutrition, Section of Gastroenterology, Department of Internal Medicine, Rush University Medical Center, 1725 W. Harrison, Suite 206, Chicago, IL 60612, USA.

Received: 14 April 2020 Accepted: 23 September 2020 Published online: 20 October 2020

\section{References}

1. Donia MS, Fischbach MA. Small molecules from the human microbiota. Science. 2015;349:1254766. https://doi.org/10.1126/science.1254766.

2. Bansal T, Alaniz RC, Wood TK, Jayaraman A. The bacterial signal indole increases epithelial-cell tight-junction resistance and attenuates indicators of inflammation. Proc Natl Acad Sci. 2010;107:228-33. https://doi.org/10.1073/ pnas.0906112107.

3. Schiering C, Wincent E, Metidji A, Iseppon A, Li Y, Potocnik AJ, et al. Feedback control of AHR signalling regulates intestinal immunity. Nature. 2017:542:242-5. https://doi.org/10.1038/nature21080.

4. Yano JM, Yu K, Mazmanian SK, Hsiao Correspondence EY, Donaldson GP, Shastri GG, et al. Indigenous bacteria from the gut microbiota regulate host serotonin biosynthesis. Cell. 2015;161:264-76. https://doi.org/10.1016/j.cell. 2015.02.047.

5. Bhattarai Y, Williams BB, Battaglioli EJ, Whitaker WR, Till L, Grover M, et al. Gut microbiota-produced tryptamine activates an epithelial G-proteincoupled receptor to increase colonic secretion. Cell Host Microbe. 2018;23: 775-85.e5. https://doi.org/10.1016/j.chom.2018.05.004.

6. Venkatesh M, Mukherjee S, Wang H, Li H, Sun K, Benechet AP, et al. Symbiotic bacterial metabolites regulate gastrointestinal barrier function via the xenobiotic sensor PXR and toll-like receptor 4. Immunity. 2014:41:296310. https://doi.org/10.1016/j.immuni.2014.06.014. 
7. Barker HA. Amino acid degradation by anaerobic bacteria. Annu Rev Biochem. 1981;50:23-40. https://doi.org/10.1146/annurev.bi.50.070181.000323.

8. Yvon M, Thirouin S, Rijnen L, Fromentier D, Gripon J. An aminotransferase from Lactococcus lactis initiates conversion of amino acids to cheese flavor compounds. Appl Envir Microbiol. 1997;63:414-9. https://doi.org/10.1128/ AEM.63.2.414-419.1997.

9. Nierop Groot MN, De Bont JAM. Conversion of phenylalanine to benzaldehyde initiated by an aminotransferase in Lactobacillus plantarum. Appl Environ Microbiol. 1998;64:3009-13. https://doi.org/10.1128/AEM.64.8. 3009-3013.1998.

10. Elsden $\mathrm{SR}$, Hilton MG, Waller JM. The end products of the metabolism of aromatic amino acids by clostridia. Arch Microbiol. 1976;107:283-8. https:// doi.org/10.1007/BF00425340.

11. Dodd D, Spitzer MH, Van Treuren W, Merrill BD, Hryckowian AJ, Higginbottom SK, et al. A gut bacterial pathway metabolizes aromatic amino acids into nine circulating metabolites. Nature. 2017;551:648-52. https://doi.org/10.1038/nature24661.

12. Dickert S, Pierik AJ, Buckel W. Molecular characterization of phenyllactate dehydratase and its initiator from Clostridium sporogenes. Mol Microbiol. 2002;44:49-60.

13. Dickert S, Pierik AJ, Linder D, Buckel W. The involvement of coenzyme A esters in the dehydration of (R)-phenyllactate to (E)-cinnamate by Clostridium sporogenes. Eur J Biochem. 2000;267:3874-84. https://doi.org/10. 1046/.j.1432-1327.2000.01427.x.

14. van Kessel SP, Frye AK, El-Gendy AO, Castejon M, Keshavarzian A, van Dijk G, et al. Gut bacterial tyrosine decarboxylases restrict levels of levodopa in the treatment of Parkinson's disease. Nat Commun. 2019;10:310. https://doi.org/ 10.1038/s41467-019-08294-y.

15. Maini Rekdal V, Bess EN, Bisanz JE, Turnbaugh PJ, Balskus EP. Discovery and inhibition of an interspecies gut bacterial pathway for levodopa metabolism. Science. 2019;364:eaau6323. https://doi.org/10.1126/science.aau6323.

16. Bianchine JR, Messiha FS, Hsu TH. Peripheral aromatic L-amino acids decarboxylase inhibitor in parkinsonism. II. Effect on metabolism of L-2- 14 C-dopa. Clin Pharmacol Ther. 1972;13:584-94. https://doi.org/10.1002/ cpt1972134584.

17. Morgan JP. Metabolism of levodopa in patients with Parkinson's disease. Arch Neurol. 1971;25:39. https://doi.org/10.1001/archneur.1971. 00490010049007.

18. Sasahara K, Nitanai T, Habara T, Kojima T, Kawahara Y, Morioka T, et al. Dosage form design for improvement of bioavailability of levodopa IV: possible causes of low bioavailability of oral levodopa in dogs. J Pharm Sci. 1981;70:730-3. https://doi.org/10.1002/jps.2600700705.

19. Goldin BR, Peppercorn MA, Goldman P. Contributions of host and intestinal microflora in the metabolism of $\mathrm{L}$-dopa by the rat. J Pharmacol Exp Ther. 1973;186:160-6.

20. Fasano A, Visanji NP, Liu LWC, Lang AE, Pfeiffer RF. Gastrointestinal dysfunction in Parkinson's disease. Lancet Neurol. 2015;14:625-39. https:// doi.org/10.1016/S1474-4422(15)00007-1.

21. Panagamuwa B, Kumar D, Ortiz J, Keighley MRB. Motor abnormalities in the terminal ileum of patients with chronic idiopathic constipation. $\mathrm{Br}$ J Surg. 1994:81:1685-8. https://doi.org/10.1002/bjs.1800811142

22. Van Der Sijp JRM, Kamm MA, Nightingale JMD, Britton KE, Granowska M, Mather SJ, et al. Disturbed gastric and small bowel transit in severe idiopathic constipation. Dig Dis Sci. 1993;38:837-44. https://doi.org/10.1007/BF01295909.

23. Dutkiewicz J, Szlufik S, Nieciecki M, Charzyńska I, Królicki L, Smektała P, et al. Small intestine dysfunction in Parkinson's disease. J Neural Transm. 2015; 122:1659-61. https://doi.org/10.1007/s00702-015-1442-0.

24. Knudsen K, Haase AM, Fedorova TD, Bekker AC, Ostergaard K, Krogh K, et al. Gastrointestinal transit time in Parkinson's disease using a magnetic tracking system. J Parkinsons Dis. 2017;7:471-9. https://doi. org/10.3233/JPD-171131.

25. Das YT, Bagchi M, Bagchi D, Preuss HG. Safety of 5-hydroxy-Ltryptophan. Toxicol Lett. 2004;150:111-22. https://doi.org/10.1016/j.toxlet. 2003.12.070.

26. Heap JT, Kuehne SA, Ehsaan M, Cartman ST, Cooksley CM, Scott JC, et al. The ClosTron: mutagenesis in Clostridium refined and streamlined. J Microbiol Methods. 2010;80:49-55. https://doi.org/10.1016/.jmimet.2009.10.018.

27. Roager HM, Hansen LBS, Bahl Ml, Frandsen HL, Carvalho V, Gøbel RJ, et al. Colonic transit time is related to bacterial metabolism and mucosal turnover in the gut. Nat Microbiol. 2016;1:16093. https://doi.org/10.1038/ nmicrobiol.2016.93.
28. Iwamoto K, Watanabe J, Yamada M, Atsumi F, Matsushita T. Effect of age on gastrointestinal and hepatic first-pass effects of levodopa in rats. J Pharm Pharmacol. 1987;39:421-5. https://doi.org/10.1111/j.2042-7158.1987.tb03413.x.

29. Zoetendal EG, Raes J, van den Bogert B, Arumugam M, Booijink CC, Troost $\mathrm{FJ}$, et al. The human small intestinal microbiota is driven by rapid uptake and conversion of simple carbohydrates. ISME J. 2012;6:1415-26. https://doi. org/10.1038/ismej.2011.212.

30. Booijink CCGM, El-Aidy S, Rajilić-Stojanović M, Heilig HGHJ, Troost FJ, Smidt $\mathrm{H}$, et al. High temporal and inter-individual variation detected in the human ileal microbiota. Environ Microbiol. 2010;12:3213-27. https://doi.org/10.1111/ j.1462-2920.2010.02294.x.

31. Costa M, Brookes SJ, Hennig GW. Anatomy and physiology of the enteric nervous system. Gut. 2000;47(Suppl 4):iv15-9; discussion iv26. https://doi. org/10.1136/gut.47.suppl_4.iv15.

32. Ringdahl B. Dissociation constants and relative efficacies of acetylcholine, (+)- and (-)-methacholine at muscarinic receptors in the guinea-pig ileum. Br J Pharmacol. 1986;89:7-13. https://doi.org/10.1111/j.1476-5381.1986. tb11115.x.

33. Jenner AM, Rafter J, Halliwell B. Human fecal water content of phenolics: the extent of colonic exposure to aromatic compounds. Free Radic Biol Med. 2005;38:763-72. https://doi.org/10.1016/j.freeradbiomed.2004.11.020.

34. Keshavarzian A, Green SJ, Engen PA, Voigt RM, Naqib A, Forsyth CB, et al. Colonic bacterial composition in Parkinson's disease. Mov Disord. 2015;30: 1351-60. https://doi.org/10.1002/mds.26307.

35. Jin JS, Hattori M. Isolation and characterization of a human intestinal bacterium Eggerthella sp. CAT-1 capable of cleaving the C-Ring of $(+)$-catechin and (-)-Epicatechin, followed by p-dehydroxylation of the Bring. Biol Pharm Bull. 2012;35:2252-6.

36. Haiser HJ, Gootenberg DB, Chatman K, Sirasani G, Balskus EP, Turnbaugh PJ. Predicting and manipulating cardiac drug inactivation by the human gut bacterium Eggerthella lenta. Science. 2013;341:295-8. https://doi.org/10. 1126/science.1235872.

37. Kalia VC, Mukherjee T, Bhushan A, Joshi J, Shankar P, Huma N. Analysis of the unexplored features of rrs (16S rDNA) of the genus Clostridium. BMC Genomics. 2011;12. https://doi.org/10.1186/1471-2164-12-18.

38. Niehues M, Hensel A. In-vitro interaction of L-dopa with bacterial adhesins of Helicobacter pylori: an explanation for clinicial differences in bioavailability? J Pharm Pharmacol. 2009;61:1303-7. https://doi.org/10.1211/jpp/61.10.0005.

39. Zimmermann M, Zimmermann-Kogadeeva M, Wegmann R, Goodman AL. Separating host and microbiome contributions to drug pharmacokinetics and toxicity. Science. 2019;363:eaat9931. https://doi.org/10.1126/science. aat9931.

40. Williams BB, Van Benschoten AH, Cimermancic P, Donia MS, Zimmermann $M$, Taketani $M$, et al. Discovery and characterization of gut microbiota decarboxylases that can produce the neurotransmitter tryptamine. Cell Host Microbe. 2014;16:495-503. https://doi.org/10.1016/j.chom.2014.09.001.

41. Lucchelli A, Boselli C, Grana E. Dopamine-induced relaxation of the guinea-pig isolated jejunum is not mediated through dopamine receptors. Pharmacol Res. 1990;22:433-44. https://doi.org/10.1016/1043-6618(90)90750-8.

42. Heap JT, Pennington OJ, Cartman ST, Carter GP, Minton NP. The ClosTron: a universal gene knock-out system for the genus Clostridium 2007;70:452-464. doi:https://doi.org/10.1016/..mimet.2007.05.021.

43. Heap JT, Pennington OJ, Cartman ST, Minton NP. A modular system for Clostridium shuttle plasmids. J Microbiol Methods. 2009;78:79-85. https://doi. org/10.1016/j.mimet.2009.05.004.

44. Williams DR, Young DI, Young M. Conjugative plasmid transfer from Escherichia coli to Clostridium acetobutylicum. J Gen Microbiol. 1990;136:81926. https://doi.org/10.1099/00221287-136-5-819.

45. Nagatsu T, Kojima K. Application of electrochemical detection in highperformance liquid chromatography to the assay of biologically active compounds. TrAC Trends Anal Chem. 1988;7:21-7. https://doi.org/10.1016/ 0165-9936(88)80026-8.

46. Wood DE, Lu J, Langmead B. Improved metagenomic analysis with Kraken 2. Genome Biol. 2019;20:1-13. https://doi.org/10.1186/s13059-019-1891-0.

47. Lu J, Breitwieser FP, Thielen P, Salzberg SL. Bracken: estimating species abundance in metagenomics data. PeerJ Comput Sci. 2017;2017:1-17. https://doi.org/10.7717/peerj-cs.104.

\section{Publisher's Note}

Springer Nature remains neutral with regard to jurisdictional claims in published maps and institutional affiliations. 